\title{
The MUSE Method for Usability Engineering
}

\author{
Kee Yong LIM \\ School of Mechanical \& Production Engineering \\ Nanyang Technological University \\ Nanyang Avenue \\ SINGAPORE 639798 \\ mkylim@ntuvax.ntu.ac.sg \\ John Brian LONG \\ Ergonomics Unit \\ University College London \\ 26 Bedford Way, London WC1H OAP, UK. \\ j.long@ucl.ac.uk
}

\begin{abstract}
Human factors input to system development is often limited to evaluation following system implementation. Since design specifications are now 'frozen' and resistent to change, human factors recruitment at this stage would predispose its recommendations toward costly design modifications. Complete implementation of its design recommendations often become impossible. The human factors input is thus 'toolittle' and 'too-late'.

One solution to the problem is to integrate structured human factors and software engineering methods. In this way, human factors inputs may be set appropriately against the system development context. To this end, a structured human factors method named MUSE (Method for USability Engineering), was developed in 1990. The objective of this tutorial is to provide participants a guided application of MUSE using design casestudies and exercises.
\end{abstract}

KEYWORDS System Development; User Interface Specification; Structured Analysis and Design Methods; Structured Human Factors Method; User Requirements and Task Analysis.

Human-Computer Interaction: INTERACT'97 S. Howard, J. Hammond \& G. Lindgaard (editors)

Published by Chapman \& Hall OIFIP 1997 


\section{OBJECTIVE OF TUTORIAL}

This full-day tutorial will demonstrate how human factors design may be supported effectively using a structured human factors method named MUSE. Participants will be provided a guided application of MUSE in design case-studies and group exercises.

\section{PARTICIPANTS TARGETED}

This tutorial is intended for human factors designers interested in wider involvement in system development, and for software engineers who wish to understand human factors design. The tutorial is intermediate in level and is targeted at experienced participants.

\section{OVERVIEW OF TUTORIAL}

The tutorial will address the following: the case for wider human factors involvement in system development; the inadequacies of rapid prototyping for facilitating timely and efficient human factors input; the optimization of human factors input by integrating MUSE with similarly structured software engineering methods; a guided tour of MUSE covering requirements and task analysis to user interface specification. The MUSE method is presented and illustrated using casestudy examples drawn from its development. Practical exercises will reinforce participants' learning of the design descriptions, procedures and notations of MUSE.

The tutorial is presented in eight units of approximately equal duration. The design concerns addressed comprise the following :

- The Case for Structured Human Factors Methods. This tutorial unit will inform participants on the advantages and disadvantages of structured methods. Contrasts with the rapid prototyping approach will be highlighted; including an account of why rapid prototyping alone is not sufficient for ensuring the development of usable human-computer systems.

- Overview of MUSE. Each stage of the method will be introduced generally and related to current practices of human factors design. In addition, relationships between
MUSE and current software engineering design activities will be highlighted.

- Detailed Tour of MUSE Part I : User Requirements and Task Analysis. Participants will be shown how different classes of existing systems (both manual and computerised) may be analysed; and how task descriptions, generalised task models, and domain descriptions, may be derived to support user requirements specification and conceptual design. The human factors design products, processes and notations involved will be illustrated using case-studies.

- Group Exercise I. Participants will be guided on how a task description may be derived for a simple case-study system. Descriptions generated by participants will then be discussed as a group, to highlight how the descriptions could support human factors evaluation and the specification of user requirements.

- Detailed Tour of MUSE Part II : Conceptual and Detailed Task Design. This tutorial unit addresses how human factors products derived at earlier stages of the method, are used to support function allocation and interactive task design.

- Detailed Tour of MUSE Part III : User Interface Specification. A user-task approach for user interface specification will be introduced in this part of the presentation. Specifically, the human factors design products, processes and notations entailed by user interface specification (comprising screen actuations, screen composition and layout, screen object behaviours, ctc.), are illustrated using case-studies.

- Group Exercises II and III. The first exercise will reinforce participants' understanding of how user interface specifications should be interpreted. The second exercise will allow participants to experience a "handson' application of the method. Both exercises will involve a design case-study.

- Summary and Future Developments. The main concerns of the tutorial will be summarised, and future developments of the method reviewed, e.g. the development of computer-based tools based on MUSE; the extension of MUSE to support declarative human factors knowledge such as design guidelines; wider integration of MUSE with established structured Software Engincering methods; etc. 